\title{
Comparison of host cell gene expression in cowpox, monkeypox or vaccinia virus-infected cells reveals virus-specific regulation of immune response genes
}

Daniel Bourquain ${ }^{1 \dagger}$, Piotr Wojtek Dabrowski ${ }^{1,2+}$ and Andreas Nitsche ${ }^{1 *}$

\begin{abstract}
Background: Animal-borne orthopoxviruses, like monkeypox, vaccinia and the closely related cowpox virus, are all capable of causing zoonotic infections in humans, representing a potential threat to human health. The disease caused by each virus differs in terms of symptoms and severity, but little is yet know about the reasons for these varying phenotypes. They may be explained by the unique repertoire of immune and host cell modulating factors encoded by each virus. In this study, we analysed the specific modulation of the host cell's gene expression profile by cowpox, monkeypox and vaccinia virus infection. We aimed to identify mechanisms that are either common to orthopoxvirus infection or specific to certain orthopoxvirus species, allowing a more detailed description of differences in virus-host cell interactions between individual orthopoxviruses. To this end, we analysed changes in host cell gene expression of HeLa cells in response to infection with cowpox, monkeypox and vaccinia virus, using whole-genome gene expression microarrays, and compared these to each other and to non-infected cells.
\end{abstract}

Results: Despite a dominating non-responsiveness of cellular transcription towards orthopoxvirus infection, we could identify several clusters of infection-modulated genes. These clusters are either commonly regulated by orthopoxvirus infection or are uniquely regulated by infection with a specific orthopoxvirus, with major differences being observed in immune response genes. Most noticeable was an induction of genes involved in leukocyte migration and activation in cowpox and monkeypox virus-infected cells, which was not observed following vaccinia virus infection.

Conclusion: Despite their close genetic relationship, the expression profiles induced by infection with different orthopoxviruses vary significantly. It may be speculated that these differences at the cellular level contribute to the individual characteristics of cowpox, monkeypox and vaccinia virus infections in certain host species.

Keywords: Orthopoxvirus, Cowpox virus, Vaccinia virus, Monkeypox virus, Microarray, Gene expression, Host cell response

\footnotetext{
*Correspondence: nitschea@rki.de

†Equal contributors

${ }^{1}$ Centre for Biological Threats and Special Pathogens 1, Robert Koch Institute,

Nordufer 20, Berlin 13353, Germany

Full list of author information is available at the end of the article
} 


\section{Background}

Viruses of the family Poxviridae are characterized by their large and complex virions, a double-stranded DNA genome of 130-375 kbp and the cytosol as the place of replication [1]. As a family, poxviruses are able to infect both vertebrate and invertebrate hosts. Poxviruses of vertebrates are divided into ten genera [2]. Out of these, especially the genus orthopoxvirus (OPV) contains several important pathogens of humans and animals, including some zoonotic members [3]. After eradication of variola virus (VARV) [4], the most common OPV infections are caused today by monkeypox virus (MPXV), vaccinia virus (VACV) and cowpox virus (CPXV) [3]. VACV is the prototype member of the OPV genus and the best-studied one. VACV served as vaccine during the smallpox eradication campaign and its virulence in man is generally low. However, several severe complications have been reported to occur after vaccination or laboratory-acquired exposition [5,6]. Furthermore, researchers in Brazil have been reporting several cases of naturally occurring zoonotic VACV infections since 1999 [7-10]. Similar to VACV infections, human CPXV infections of healthy individuals are generally self-limiting and cause only localised skin lesions. However, severe generalised CPXV infections with lethal outcome have been reported in immunocompromised patients $[11,12]$. In Europe and parts of northern and central Asia, endemic CPXV are the most common cause of human OPV infections [13]. It is assumed that wild rodents serve as reservoir hosts for CPXV. However, transmission to various other species including several domestic and zoo animals has been reported, and of all OPV CPXV is suggested to possibly infect the widest range of host species [3]. To date, no direct human-to-human transmission has been reported $[13,14]$. In contrast to VACV and CPXV, MPXV causes a disease resembling smallpox in humans, but with milder morbidity and lower mortality rates [15]. The severity of the disease depends on the geographic origin of the different MPXV strains, as virus isolates from Central Africa have been shown to be more virulent than those from Western Africa [16,17]. MPXV was first described as an illness of captive zoo monkeys [18], and rodents are assumed to be the natural host [3].

Concerning the potential threat arising from VACV, MPXV and CPXV, a deepening of our knowledge about the mechanisms underlying differences in poxviral pathogenesis and species-specificity would allow greatly improved risk assessment.

Today, more than 100 OPV genomes have been fully sequenced. Therefore the unique arsenal of viral genes encoded by each virus is often known, and in several cases detailed information about viral gene functions is also available. However, current knowledge about corresponding events in the host cell and especially of the differences in host response towards infection and host modulation by these viruses is still limited. Several studies described the transcriptional host response towards infection of different cell types with VACV or closely related rabbitpox virus, either using microarrays or ultrahigh-throughput DNA sequencing for genomewide transcriptome analysis [19-25]. These studies report that host genes are predominantly downregulated during infection, which may be due to an unspecific suppression of host mRNAs by VACV. The fewer cellular genes which are specifically induced by infection are supposed to play key roles in viral replication or host response to infection, respectively $[19,22,25]$. Especially mRNAs which are associated with the NF- $k B$ cascade, apoptosis, signal transduction and ligand-mediated signalling were reported to be induced in response to VACV infection [21]. Similarly, MPXV was described to cause a decrease in host mRNA levels, accompanied by an increase of fewer mRNAs following infection of MK2 cells [26]. Besides these studies, which focused on analysis of the host response to infection by one specific virus, Rubins et al. directly compared how VACV and MPXV alter the gene expression programs in their hosts [27]. However, to our knowledge, so far no study has investigated the transcriptional response of the host cell towards CPXV infection. As CPXV encodes several unique genes, not to be found in the VACV or MPXV genome, we decided to compare the way how these zoonotic poxviruses alter the gene expression of their host cells. We chose HeLa cells as a model system and characterized changes in the host transcriptional programs in response to infection with CPXV, MPXV or VACV, respectively, using microarrays representing the whole human genome. We aimed to identify mechanisms that are either common to OPV infection or to specific OPV species at the cellular level, allowing a more detailed description of differences in virus-host cell interactions between individual OPV.

\section{Results}

\section{Experimental design}

HeLa cells were infected with either CPXV reference strain Brighton Red (BR), the highly pathogenic central African MPXV strain MSF-6 [28] or the mouse-pathogenic VACV strain IHD-W [29]. HeLa cells were chosen because of their high susceptibility towards OPV infection and because many fundamental studies on OPV biology and on host gene expression changes following infection have been performed with this cell line [19-21,23,25]. Cells were infected at a high multiplicity of infection of 5 plaque forming units (PFU) per cell to guarantee synchronous infection of all cells. Total RNA from infected and mockinfected control cells was isolated at $6 \mathrm{~h}$ post infection. This point of time was chosen to allow enough time for establishment of infection and progression to late viral gene 
expression but to avoid the risk of cell lysis and RNA degradation after completion of the first replication cycle $[1,24,30]$. Furthermore, previous studies described that specific gene activation in response to infection mainly occurs until $6 \mathrm{~h}$ post infection, while unspecific downregulation of genes prevails at later stages of infection [19,23]. Therefore, we chose $6 \mathrm{~h}$ post infection to analyse the specific host cell modulation by CPXV, MPXV and VACV and to analyse differences in the cellular response towards these viruses. The successful infection of HeLa cells with all viruses and the uniform progression of infection were confirmed by immunofluorescence microscopy of cells infected in parallel. At $24 \mathrm{~h}$ post infection with each virus nearly all cells were infected and showed a pronounced cytopathic effect (data not shown). To verify that all viruses had uniformly proceeded from intermediate to late viral transcription at $6 \mathrm{~h}$ post infection, mRNA expression of the late transcription factor VLTF-1 (VACCP-G8R) and of the late $11 \mathrm{kDa}$ virion core protein (VACCP-F17R) was confirmed via quantitative real-time PCR (data not shown).

Total RNA from infected and mock-infected control cells was used for cRNA microarray analysis of host cell transcription. All experiments were performed in duplicate using RNA samples from two independently infected cell cultures for each analysis. To compare the gene expression profiles, ratios were calculated by dividing the merged normalised signal intensities of infected samples by mock-control signal intensities. Genes that exhibited a fold change (FC) in gene expression $\geq 2$ and signal intensities that were significantly above the background with p-values $\leq 0.01$ were chosen for further analysis (see also Additional file 1).

\section{The majority of host genes remains unaffected after infection}

At first, we compared the gene expression data of each virus-infected sample to the expression profile of the mock-infected HeLa cells to determine the relative modulation of cellular transcription induced by infection with CPXV, MPXV or VACV, respectively. As shown in Figure 1A, a majority of $96 \%$ of the assayed cellular transcripts remained unchanged after infection with either virus strain. Out of the 30,484 individual genes and transcripts assayed in total (using 41,000 probes), 1,027 (3.7\%) transcripts in CPXV-infected cells, only 321 (1.1\%) transcripts in MPXV-infected cells and 1,002 (3.3\%) transcripts in VACV-infected cells showed expression changes larger than 2-fold after infection. Among the 1,027 CPXV-modulated transcripts, 660 (64.3\%) were upregulated and only 367 downregulated. A similar distribution can be seen following MPXV infection which resulted in upregulation of 219 (68.2\%) and downregulation of 102 transcripts. Likewise, VACV infection resulted in upregulation of $708(70.7 \%)$ and downregulation of 294 transcripts. Taken together, these data show that an infection with CPXV, VACV and
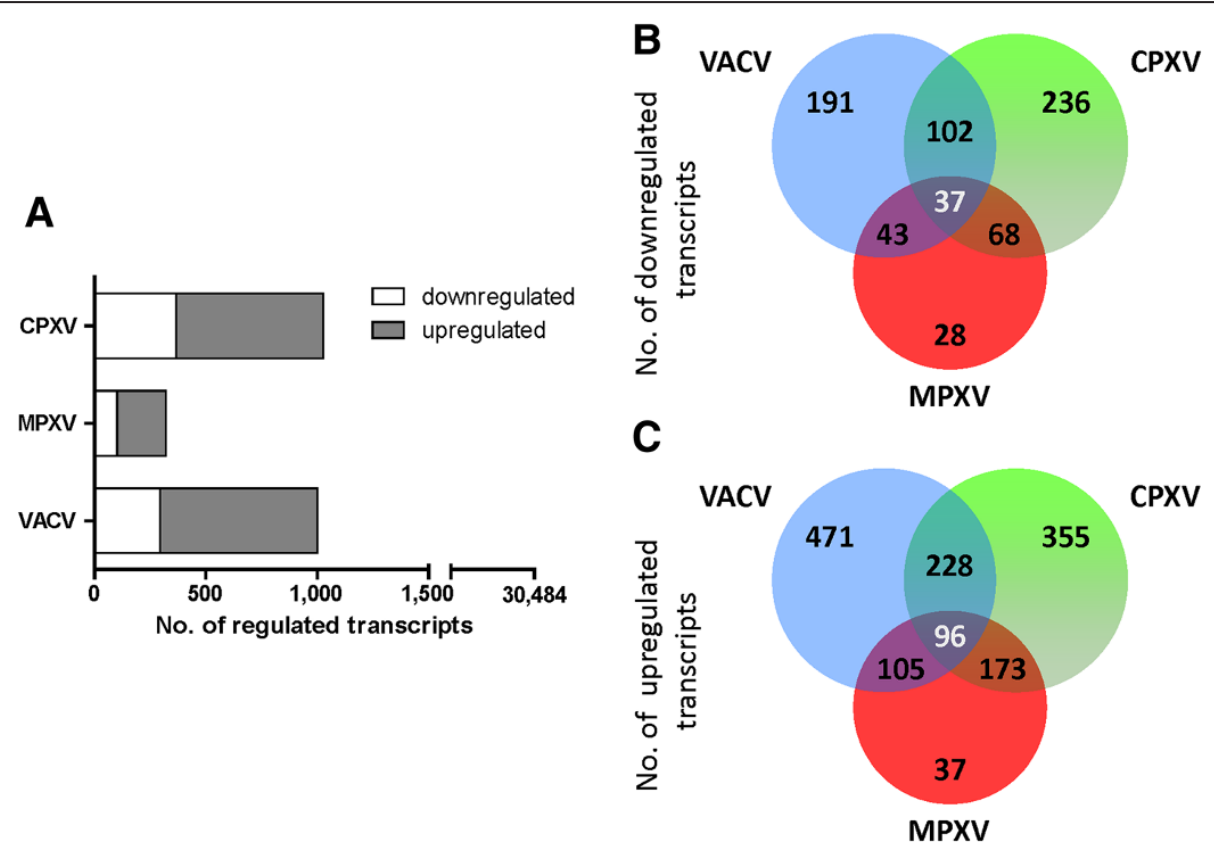

Figure 1 Number of host mRNAs regulated by CPXV, MPXV or VACV infection. Shown are the numbers of cellular transcripts that exhibited expression changes larger than 2-fold after infection with each virus in two independent microarray experiments. Transcripts that were upregulated after infection in comparison to non-infected cells are shown in dark grey, transcripts that were downregulated in white (A). Intersections of transcript downregulation (B) or upregulation (C) in response to VACV, CPXV or MPXV infection are shown in the Venn diagram. 
especially MPXV induces only marginal changes of the cellular gene expression profile at $6 \mathrm{~h}$ post infection.

\section{Identification of gene expression changes common to CPXV, MPXV and VACV infection}

Despite of the fact that the number of host cell transcripts affected by CPXV and VACV infection was similar, the number of genes regulated by CPXV and VACV was only $330(\sim 33 \%)$ of approximately 1,000. Among the 321 host transcripts which are modulated by MPXV infection, 241 (75.1\%) were also affected by CPXV but only 148 (46.1\%) by VACV infection. Beyond that, by comparing all three data sets, we could identify a cluster of 133 transcripts whose expression is modulated after infection with each virus. Out of these, 96 transcripts (72.2\%) were upregulated in all virus-infected samples and 37 were downregulated (Figure 1B, C). We applied a Gene Ontology (GO) termbased cluster analysis of these 133 transcripts to identify the biological processes in which the genes are involved. Analysis of gene set enrichment was used to identify statistically overrepresented processes. This analysis revealed that the most prominent group were genes involved in chromatin organisation, containing 50 of the 133 transcripts. Out of these, 46 belonged to the histone family of proteins. Increased detection of histone mRNAs following OPV infection has been described before to be an experimental artefact caused by de novo polyadenylation of these mRNAs by the viral poly(A) polymerase, resulting in their enhanced amplification and detection [22,27]. Therefore histone mRNAs were excluded from further analysis, resulting in 87 genes which were regulated by infection with CPXV, MPXV as well as VACV. A comparison of the gene expression of these genes is shown in Additional file 2 . Besides the histone family members, further functional clusters of transcripts were identified.

\section{Analysis of commonly regulated genes reveals an induction of epidermal growth factor family members and genes involved in regulation of MAPK activity by CPXV, MPXV and VACV}

Cluster analysis of the 87 non-histone transcripts that were affected by all OPV tested revealed a statistically significant (Bonferroni-corrected group p-value $=1.1 \times 10^{-5}$ ) overrepresentation of genes involved in inactivation of mitogen-activated protein kinase (MAPK) activity (DUSP5/ 6 , SPRED1/2, SPRY2/4). All of these genes were upregulated in virus-infected cells and all of them are known to be induced following MAPK-ERK activation [31-35]. In this context, we could also observe a strong upregulation of the early growth-response gene EGR1 and a less pronounced upregulation of EGR2 by all three viruses. EGR1 upregulation via the MAPK-ERK pathway following
VACV and CPXV infection has been described before by Silva et al. [36]. Here we show a similar effect following MPXV infection as well. To further evaluate the general impact of OPV infection on biological processes of the infected cell, we examined potential enrichment of these 87 commonly affected genes in known canonical pathways, according to the KEGG pathway database. We could identify $12 / 87$ genes which significantly mapped to two distinct pathways, the ErbB pathway with $5.75 \%$ of associated genes (AREG, CBLB, EREG, HBEGF, PRKCB; $\mathrm{p}=1.6 \times 10^{-3}$ ) and the JAK-STAT pathway with $4.52 \%$ of associated genes (CBLB, IL4R, LIF, SPRED1/2, SPRY2/4; $\mathrm{p}=2.1 \times 10^{-4}$ ). The ErbB signalling pathway comprises a family of receptor tyrosine kinases which are coupling the binding of extracellular growth factor ligands to intracellular signalling pathways such as the MAPK pathway. In this context, upregulation of the epidermal growth factor (EGF) family members EREG, AREG and HBEGF, respectively, may be seen as a trigger for enhanced MAPK-ERK signalling which itself results in upregulation of DUSP5/6, SPRED1/2, SPRY2/4 and EGR1/2, respectively, as a feedback mechanism. The induction of EGF family members and genes involved in regulation of MAPK activity by VACV, CPXV as well as MPXV is summarized in Table 1. Interestingly, only the immune-regulatory CBLB gene and the multifunctional PRKCB gene were downregulated by infection.

\section{Identification of virus-specific changes in gene expression induced by CPXV, MPXV or VACV infection}

In contrast to these few commonly regulated genes, most infection-regulated genes differ from virus to virus. These differences were most prominent when comparing CPXV or MPXV to VACV infection. To this end, we attributed the enrichment of all genes to biological processes that were differentially regulated following infection in comparison to non-infected cells. This analysis again shows the common regulation of the MAP kinase pathway by all three viruses, but also highlights major differences in the gene clusters affected by CPXV, MPXV or VACV infection. All results are summarized in Table 2. In the case of CPXV infection we could identify 16 overrepresented clusters, including several genes encoding protein kinases, proteins mediating kinase activity and blood vessel development. However, this cluster also contained a significant overrepresentation of genes which can be involved in immune response-activating signal transduction such as Toll-like receptor signalling pathways. Notably, other significantly overrepresented groups were associated with immune system processes, too, such as regulation of leukocyte migration and myeloid cell differentiation.

In the case of MPXV infection again an enrichment of genes involved in negative regulation of MAPK 


\begin{tabular}{|c|c|c|c|c|}
\hline & \multirow[t]{2}{*}{ Gene } & \multicolumn{3}{|c|}{ Gene expression changes (average FC) } \\
\hline & & CPXV & MPXV & VACV \\
\hline \multirow[t]{11}{*}{ MAPK activity } & DUSP5 & 6.6 & 4.9 & 3.6 \\
\hline & DUSP6 & 8.1 & 9.5 & 5.2 \\
\hline & EGR1 & 14.3 & 30.8 & 98.6 \\
\hline & EGR2 & 2.6 & 5.1 & 4.2 \\
\hline & $\| \mathrm{L} 4 \mathrm{R}$ & 6.2 & 5.8 & 2.9 \\
\hline & LIF & 9.7 & 12.6 & 4.6 \\
\hline & SPRY1 & 12.7 & n.s. ${ }^{b}$ & n.s. ${ }^{b}$ \\
\hline & SPRY2 & 7.7 & 7.1 & 7.0 \\
\hline & SPRY4 & 20.8 & 30.1 & 11.8 \\
\hline & SPRED1 & $2.8 / 3.7^{c}$ & $2.9 / 3.0^{c}$ & $2.2 / 2.2^{c}$ \\
\hline & SPRED2 & 2.0 & 2.3 & $2.8 / 7.8^{c}$ \\
\hline both & CBLB & -2.7 & -3.4 & -2.5 \\
\hline \multirow[t]{4}{*}{ ErbB pathway } & AREG & 10.2 & 8.3 & 4.8 \\
\hline & EREG & $4.4 / 6.7^{c}$ & $7.0 / 10.3^{c}$ & $2.9 / 3.0^{c}$ \\
\hline & HBEGF & $4.0 / 5.4^{c}$ & $2.3 / 2.6^{c}$ & $2.1 / 2.8^{c}$ \\
\hline & PRKCB & -4.9 & -3.4 & -2.4 \\
\hline
\end{tabular}

${ }^{a}$ Cluster and pathway analysis of the 87 non-histone transcripts that were affected by all OPV tested revealed a statistically significant overrepresentation of genes involved in inactivation of mitogen-activated protein kinase (MAPK) activity and growth factor signalling. Shown are gene expression changes of the identified genes at $6 \mathrm{~h}$ post infection in CPXV-, VACV- or MPXV-

infected cells in comparison to mock-infected cells as average fold change (FC) values.

${ }^{b}$ Values that did not meet the analysis cut-off of an average $F C \geq 2.0$ and $\mathrm{p} \leq 0.05$ are designated n.s. (not significant).

' Two values separated by a slash designate values obtained from two different probes on the microarray.

activity and negative regulation of intracellular protein kinase cascade was observed. Similar to CPXV infection, immunity-associated clusters were likewise overrepresented. This included the biological processes "cellular response to lipopolysaccharide" and "positive regulation of NF-kB transcription factor activity". Many of the regulated genes within either of these processes are also involved in positive regulation of leukocyte migration, Toll-like receptor signalling and chemotaxis.

In contrast, host genes modulated by VACV infection showed a significant overrepresentation only in four Gene Ontology (GO) terms. The most significantly affected biological process was negative regulation of intracellular protein kinase cascade and especially of the MAPK cascade. However, in comparison to CPXV and MPXV infection, no immune response-specific processes could be identified. Taken together, we could identify considerable differences concerning the expression of immunity-associated host genes which were significantly affected by CPXV and MPXV but not by VACV infection.
Analysis of canonical pathways reveals a significant influence of CPXV and MPXV but not VACV infection on cellular pathways involved in the immune response and infectious diseases

Infection with CPXV influenced the expression of 1,027 genes, 244 of which were represented in the KEGG pathway database, and 126 of these genes could be mapped to 21 significantly affected pathways (Figure 2). In the case of MPXV infection 104 genes were found in KEGG, and 35 of them could be mapped to pathways that met the criteria. Both after CPXV and MPXV infection a noticeable overrepresentation of immunity-related pathways was observed, along with a significant overrepresentation of genes mapping to pathways specific for certain infectious diseases. Interestingly, for the most part the diseasespecific pathways which were affected by CPXV and MPXV infection describe infections caused by intracellular pathogens or inflammatory processes. For VACV-infected cells 191 out of 949 genes were found in KEGG. However, in sharp contrast to the numerous cellular pathways affected by CPXV or MPXV infection, only the ErbB signalling pathway was significantly affected by VACV infection. All significantly affected pathways are shown in Figure 2.

Taken together, this shows the general importance of growth factor and MAPK signalling for OPV infection. Furthermore, the analysis highlighted that immune responseassociated signalling pathways are highly affected by CPXV and MPXV, but not by VACV infection. Several genes which are affected by CPXV and MPXV infection were involved in disease-specific pathways. However, the majority of those genes also mapped to other, less pathogen-specific pathways like Toll-like and NOD-like receptor signalling pathways, phagosome, MAPK signalling and apoptosis pathways, albeit with a less significant enrichment.

\section{CPXV and MPXV modulate genes involved in leukocyte migration and TLR signalling}

We analysed enrichment of genes involved in immune system processes to further specify these results. To this end, we matched these genes against the GO immune system process database. We could identify an overrepresentation of genes involved in positive regulation of leukocyte migration $\left(\mathrm{p}=1.2 \times 10^{-2}, 21.8 \%\right)$ and Toll-like receptor 2 signalling pathways $\left(\mathrm{p}=4.9 \times 10^{-2}\right.$, $18.1 \%)$ after CPXV infection, and in positive regulation of leukocyte migration $\left(\mathrm{p}=6 \times 10^{-3}, 12.7 \%\right)$ and TRIF-dependent Toll-like receptor signalling pathways $\left(\mathrm{p}=5.9 \times 10^{-3}, 11.3 \%\right)$ after MPXV infection. No such overrepresentation could be detected among genes that showed regulation following VACV infection (data not shown). 
Table 2 Cluster analysis of infection-induced gene expression changes

\begin{tabular}{|c|c|c|c|c|}
\hline & Leading term $^{a}$ & No. of genes ${ }^{b}$ & Group p-value & Term $p$-value $^{b}$ \\
\hline \multirow[t]{17}{*}{$\overline{C P X V}$} & blood vessel development & 49 & $4.8 \times 10^{-11}$ & $7.6 \times 10^{-9}$ \\
\hline & negative regulation of protein kinase activity & 25 & $1.4 \times 10^{-14}$ & $1.1 \times 10^{-8}$ \\
\hline & myeloid cell differentiation & 22 & $6.5 \times 10^{-4}$ & $5.4 \times 10^{-3}$ \\
\hline & negative regulation of protein serine/threonine kinase activity & 22 & $8.0 \times 10^{-18}$ & $1.1 \times 10^{-10}$ \\
\hline & ERK1 and ERK2 cascade & 20 & $1.7 \times 10^{-11}$ & $3.8 \times 10^{-6}$ \\
\hline & regulation of ERK1 and ERK2 cascade & 19 & $1.8 \times 10^{-12}$ & $1.3 \times 10^{-6}$ \\
\hline & regulation of actin cytoskeleton organization & 18 & $1.1 \times 10^{-4}$ & $4.5 \times 10^{-3}$ \\
\hline & negative regulation of MAP kinase activity (gene set 1) & 17 & $4.4 \times 10^{-8}$ & $1.0 \times 10^{-8}$ \\
\hline & negative regulation of MAP kinase activity (gene set 2) & 17 & $1.4 \times 10^{-11}$ & $1.0 \times 10^{-8}$ \\
\hline & regulation of leukocyte migration & 14 & $6.2 \times 10^{-8}$ & $5.5 \times 10^{-5}$ \\
\hline & negative regulation of hormone secretion & 13 & $4.6 \times 10^{-5}$ & $1.0 \times 10^{-6}$ \\
\hline & negative regulation of Wnt receptor signalling pathway & 11 & $4.2 \times 10^{-3}$ & $1.1 \times 10^{-1}$ \\
\hline & nephron development & 11 & $2.4 \times 10^{-5}$ & $3.8 \times 10^{-2}$ \\
\hline & positive regulation of nitric oxide biosynthetic process & 10 & $5.0 \times 10^{-6}$ & $2.5 \times 10^{-5}$ \\
\hline & positive regulation of transmission of nerve impulse & 9 & $7.7 \times 10^{-4}$ & $3.9 \times 10^{-3}$ \\
\hline & positive regulation of mitosis & 7 & $2.2 \times 10^{-6}$ & $1.8 \times 10^{-2}$ \\
\hline & negative regulation of insulin receptor signalling pathway & 6 & n.a. ${ }^{c}$ & $1.3 \times 10^{-2}$ \\
\hline \multirow[t]{8}{*}{ MPXV } & positive regulation of NF-KB transcription factor activity & 12 & $1.8 \times 10^{-16}$ & $5.3 \times 10^{-8}$ \\
\hline & negative regulation of intracellular protein kinase cascade & 11 & п.а. ${ }^{c}$ & $2.6 \times 10^{-6}$ \\
\hline & negative regulation of hormone secretion & 10 & n.a. ${ }^{c}$ & $3.3 \times 10^{-9}$ \\
\hline & negative regulation of MAP kinase activity & 8 & n.a. ${ }^{c}$ & $1.8 \times 10^{-5}$ \\
\hline & cellular response to lipopolysaccharide & 8 & $9.3 \times 10^{-9}$ & $8.1 \times 10^{-5}$ \\
\hline & positive regulation of Rho GTPase activity & 7 & n.a. ${ }^{c}$ & $2.7 \times 10^{-4}$ \\
\hline & astrocyte differentiation & 6 & n.a. ${ }^{c}$ & $2.0 \times 10^{-4}$ \\
\hline & regulation of gliogenesis & 6 & n.a. ${ }^{c}$ & $1.3 \times 10^{-3}$ \\
\hline \multirow[t]{4}{*}{ VACV } & negative regulation of MAPK cascade & 12 & $7.0 \times 10^{-6}$ & $2.5 \times 10^{-4}$ \\
\hline & ovum-producing ovary development & 8 & n.a. ${ }^{c}$ & $4.8 \times 10^{-2}$ \\
\hline & potassium ion transmembrane transport & 6 & n.a. ${ }^{c}$ & $4.1 \times 10^{-2}$ \\
\hline & cellular potassium ion transport & 6 & n.a. ${ }^{c}$ & $4.1 \times 10^{-2}$ \\
\hline
\end{tabular}

a Overrepresented biological processes identified by GO clustering of the genes affected by CPXV, MPXV or VACV infection. Related terms were merged into functional groups to reduce redundancy, and the most significant term of the group was defined as group-leading term.

${ }^{\mathrm{b}}$ The degree of connectivity between terms is calculated using $\mathrm{k}$ statistics, and the calculated $\mathrm{k}$ score is also used for defining functional groups. Shown are the identified overrepresented processes, the number of infection-regulated genes assigned to that process and the term $p$-values.

${ }^{c}$ n.a. $=$ not applicable. Only term p-values are given if no grouping was done.

\section{CPXV and MPXV strongly induce genes involved in chemotaxis or leukocyte activation}

A comparison of the changes in the host gene expression of immune response-affiliated genes, as defined by respective GO terms, showed several genes which were highly upregulated after CPXV or MPXV but not or only slightly upregulated after VACV infection. Especially following CPXV infection an upregulation of several inflammatory genes was observed. Furthermore, several genes encoding proteins which possess chemokine activity or are involved in positive regulation of leukocyte migration were induced by CPXV infection. This upregulation was similar for MPXV infection, but not or only weakly present following VACV infection. Additionally, we could observe a pronounced upregulation of several genes involved in leukocyte activation and positive regulation of leukocyte activation following CPXV infection, which could not be observed following VACV infection and only to a lesser extent following MPXV infection (Figure 3). The numbers of up- and downregulated genes which are associated with these GO terms are also shown in Table 3.

Taken together, these data indicate that in contrast to VACV infection the regulation of immune responseassociated genes by CPXV and MPXV infection may more likely indicate a pronounced antiviral immune response of the host cell than a process beneficial for viral replication. 

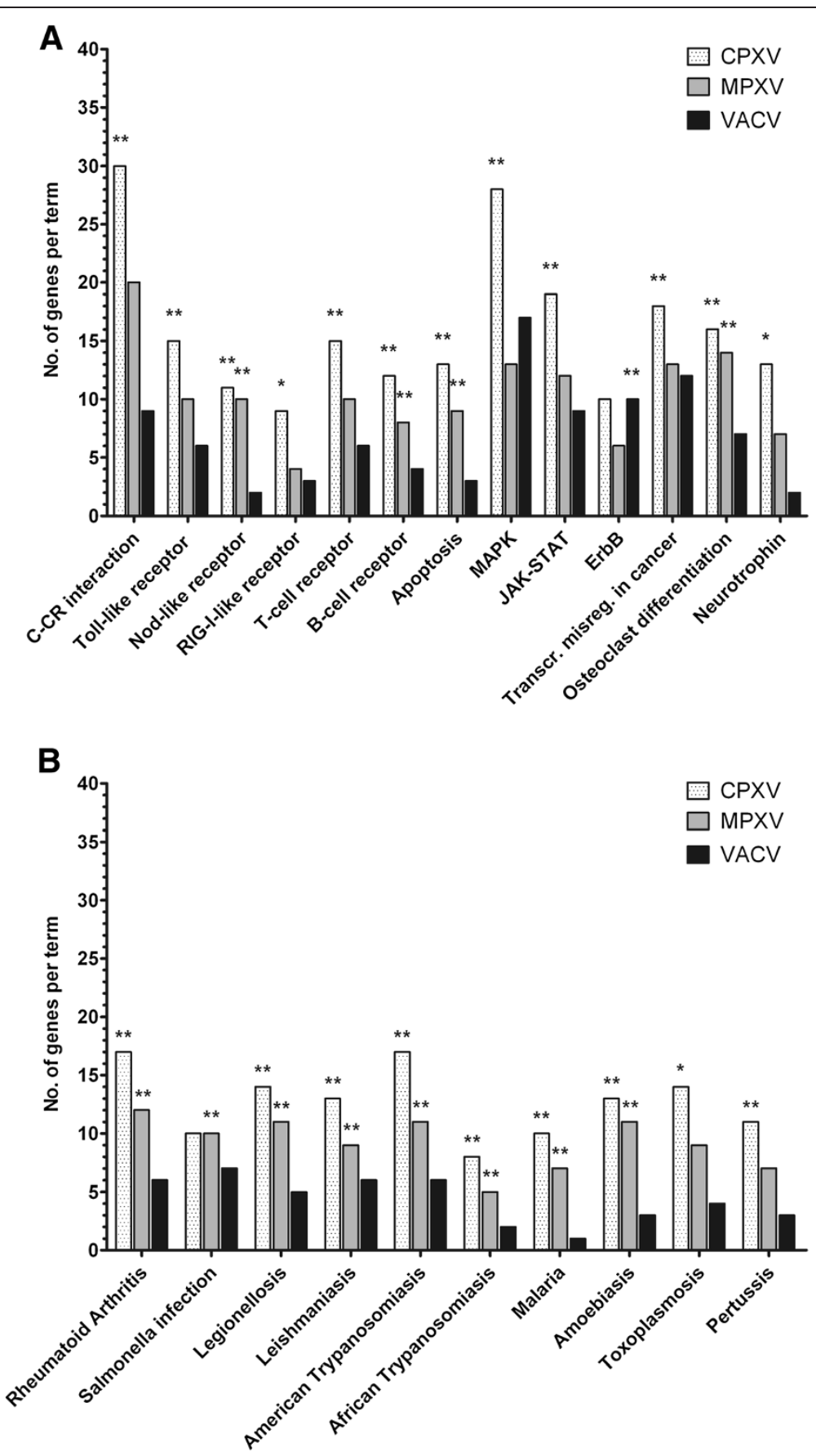

Figure 2 CPXV- and MPXV- but not VACV-modulated cellular pathways are involved in infectious diseases. Cellular pathways that were significantly affected by infection with CPXV, MPXV or VACV, respectively, as indicated by* $(p \leq 0.05)$ or ** $(p \leq 0.01)$. Along with pathways which describe general processes of the cell, we identified several immune system-specific pathways $(\mathbf{A})$ and several pathways which are specific to certain diseases (B). P-values indicate the probability of random association between the genes in the data set and the canonical pathway. Abbreviations used in the figure: C-CR interaction = cytokine-cytokine receptor interaction; Transcr. misreg. in cancer = transcriptional misregulation in cancer. 


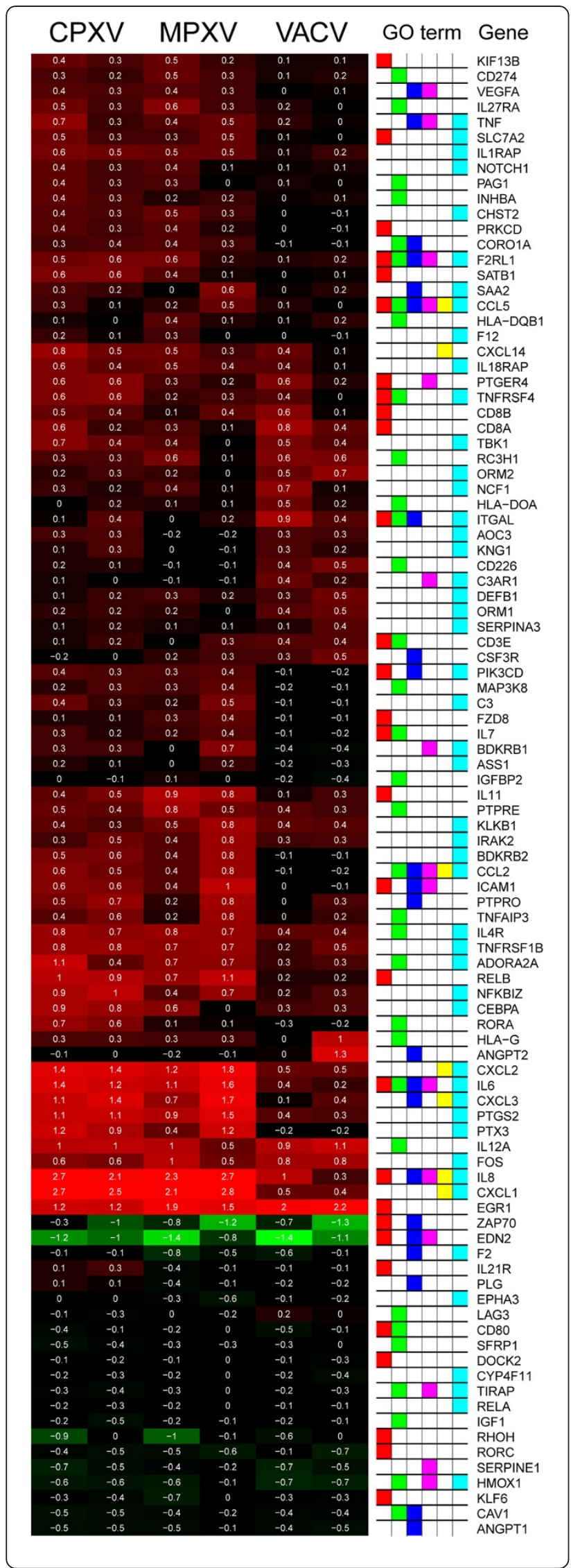

Figure $\mathbf{3}$ Modulation of immune response-associated genes by CPXV, MPXV or VACV infection. An overview of the changes in host gene expression patterns of immune response-associated genes induced by all three different OPV. Folds of change in gene expression in comparison to mock-infected cells are represented as gradient red and green colour representing low and high relative expression in the respective microarray (shown are 2 arrays per virus). Numbers indicate the $\log 10$ of the change fold values. GO term assignment of the respective genes is displayed by different colours. Red = "leukocyte activation", green = "regulation of leukocyte activation", blue = "leukocyte migration", magenta = "regulation of leukocyte migration", yellow = "human chemokine activity", turquoise = "inflammatory response". Only those genes are shown which had at least a 2-fold change in expression relative to the mock-infected control and for which the direction of regulation detected by both arrays was the same. Additionally, regulation in each selected gene differs by a factor of at least 2 between at least two of the samples.

This is supported by the observed upregulation of many pro-inflammatory genes and especially chemokine genes following CPXV and MPXV infection.

\section{Discussion}

In this study we applied a comparative analysis of changes of the host cell gene expression profile following infection with CPXV, MPXV or VACV, respectively. We addressed the question whether the different characteristics of each virus, especially the distinctive repertoire of host modulating factors encoded by each virus, may in part be reflected by different characteristics of the infected cells. OPV are well known to suppress the antiviral host defence, exploit the host cell machinery for reproduction and to inhibit or delay cell death. However, most of our knowledge about these mechanisms originates from studies using VACV. More recently, after the US outbreak in 2003, MPXV has come into focus $[37,38]$, but again, our knowledge about the differences in virus-host interactions between VACV and MPXV and even more so between VACV, MPXV and CPXV is still limited. In this study we investigated the hostcell transcriptome of CPXV-, MPXV- or VACV-infected cells to explore the different capabilities of each virus to interact with the host cell.

We found the host cell transcriptome to be mainly unaffected by poxviral infection, despite the major morphological changes induced by infection. Although this holds true for OPV infection in general, the impact of MPXV infection on the transcriptome seems to be uniquely low. This might indicate an even more elaborate "stealth" program than that performed by VACV or CPXV, allowing MPXV to especially well avoid the responses of the innate and adaptive immune systems to the developing infection. It would be interesting to investigate if this is true for MPXV infection in general, or if it might be a characteristic of the highly pathogenic central African MPXV strains in contrast to less pathogenic west African strains [17]. 
Table 3 Numbers of at least 2-fold up- or downregulated immune response-associated genes following CPXV, MPXV or VACV infection grouped by associated GO term

\begin{tabular}{|c|c|c|c|c|c|c|c|c|c|c|c|c|}
\hline & \multicolumn{2}{|c|}{$\begin{array}{l}\text { Leukocyte } \\
\text { activation }\end{array}$} & \multicolumn{2}{|c|}{$\begin{array}{c}\text { Regulation of leukocyte } \\
\text { activation }\end{array}$} & \multicolumn{2}{|c|}{$\begin{array}{l}\text { Leukocyte } \\
\text { migration }\end{array}$} & \multicolumn{2}{|c|}{$\begin{array}{c}\text { Regulation of leukocyte } \\
\text { migration }\end{array}$} & \multicolumn{2}{|c|}{$\begin{array}{c}\text { Human chemokine } \\
\text { activity }\end{array}$} & \multicolumn{2}{|c|}{$\begin{array}{l}\text { Inflammatory } \\
\text { response }\end{array}$} \\
\hline & $\overline{U p}$ & Down & Up & Down & Up & Down & Up & Down & Up & Down & Up & Down \\
\hline $\mathrm{CPXV}$ & 17 & 6 & 15 & 6 & 12 & 4 & 8 & 4 & 6 & 0 & 29 & 2 \\
\hline MPXV & 16 & 6 & 16 & 2 & 13 & 6 & 9 & 3 & 7 & 0 & 31 & 3 \\
\hline VACV & 9 & 5 & 11 & 4 & 6 & 5 & 4 & 4 & 5 & 0 & 20 & 4 \\
\hline
\end{tabular}

The data presented are in agreement with the results by Rubins et al. which show no general decline in cellular mRNAs after infection of HeLa cells with MPXV (Zaire strain) or VACV (Western Reserve strain) [27]. In our study we could prove that the same was true for CPXV infection. Other studies describe that the cellular gene expression is generally suppressed in response to infection and that only few genes are specifically induced $[19,23,26]$. In our study, we found that more than two thirds of the few host cell transcripts which exhibited regulation in response to infection were upregulated at $6 \mathrm{~h}$ post infection. However, we cannot exclude the possibility of a more pronounced downnregulation of host cell genes at later stages of infection. Furthermore, in our study, only genes were taken into account that exhibited at least 2-fold change in gene expression, to set the focus on genes that exhibit stronger regulation. Therefore, a mild global downregulation of cellular genes which was below the cut-off value might have been neglected (see also Additional file 3). However, while repression of host genes by OPV is thought to be generalised and likely nonspecific, cellular genes which are induced by infection are of particular interest, as they are thought to play key roles in viral replication or host response to infection $[19,22,25]$.

We found the infection-induced changes of the gene expression profiles of CPXV-, MPXV- and VACVinfected cells to be largely different. However, we could still identify several cellular transcripts which showed similar modulation after infection with each virus, the most significant one being histone mRNAs. An apparent induction of histone genes has been described previously in other studies [19-21,26,27]. However, this is thought to be an experimental artefact caused by de novo polyadenylation of the histone transcripts by the viral poly(A) polymerase, as histone mRNAs are the only mRNAs in eukaryotes that lack a poly(A) tail in general [22,27]. This is supported by the fact that Yang et al. reported histone mRNAs to be overrepresented at $4 \mathrm{~h}$ post VACV infection even after poly (A)-specific mRNA isolation procedures [21]. If this was the case, amplification of histone mRNAs from infected samples would be greatly enhanced by the poly(A)dependent procedure we used. We therefore decided to exclude histone mRNAs from further analysis.
After exclusion of histone mRNAs, we could identify 87 cellular transcripts which seemed to be commonly modulated by OPV infection in general. Most noticeable was an apparent infection-induced upregulation of genes involved in inactivation of MAPK activity. The upregulated Sprouty (SPRY2/4) and Sprouty-related (SPRED1/2) proteins are known to be induced by growth factor receptor activation-mediated MAPK-ERK activation via Ras as a self-regulatory feedback inhibition mechanism [31-33]. Similarly, the upregulated DUSP5/6 and EGR1/2 genes are commonly induced as early response genes after activation of the MAPK-ERK signalling pathway and act as negative regulators of ERK phosphorylation [34,35]. Therefore we suggest that the observed upregulation of genes involved in inactivation of MAPK activity may reflect a feedback mechanism towards virus-induced stimulation of MAPK-ERK activity, probably via an enhanced virus-induced signalling through EGF receptors. This theory is supported by the observed overrepresentation of components of the ErbB pathway among the commonly affected transcripts and by the upregulation of EGF family members following infection. Although modulation of the MAPK-ERK pathway seems to be a common effect of OPV infection, its importance differs from virus to virus. Silva et al. showed that inhibition of MEK/ERK signalling resulted in a significant decrease in VACV yield, but had no impact on CPXV replication $[36,39,40]$. The importance of MAPK-ERK signalling in the context of MPXV replication has not been addressed yet.

Despite these few commonly affected gene sets, major differences in the transcriptional response towards infection with CPXV, MPXV or VACV prevailed. Most interesting was an enrichment of genes involved in immunityassociated processes and pathways in CPXV- and MPXVinfected cells, which was strikingly absent following VACV infection. This included especially genes that are involved in leukocyte migration and Toll-like receptor signalling, which seemed to be affected by CPXV as well as by MPXV infection.

Interestingly, we could identify several pathways specific to certain infectious diseases to be affected by CPXV and MPXV infection. As most of these pathways were specific to infection by intracellular pathogens, this result may be explained by universal mechanisms of host modulation and 
inflammatory host response which could be affected alike by these diseases and by CPXV and MPXV.

A large proportion of the immunity-associated genes which were affected by CPXV and MPXV consisted of pro-inflammatory cytokine genes and genes involved in leukocyte chemotaxis or activation of immune cells. As most of these genes showed pronounced upregulation after CPXV and MPXV infection, this might reflect an inadequate subversion of the hosts' antiviral response. This may be supported by the fact that an induction of genes implicated in the immune response, e.g. IL6, could also be observed in response to attenuated modified VACV Ankara infection of HeLa cells [19] but not in response to non-attenuated VACV WR infection [23]. Interestingly, an induction of pro-inflammatory cytokines and chemokines in response to CPXV and MPXV infection was observed by other studies as well. Increased IL8 gene expression following MPXV infection of MK2 cells was reported by Alkhalil et al. [26]. And in vivo, strong secretion of IL-6, IL-8, and G-CSF or of IL-6, IL-8 and CCL-2, respectively, was observed following infection of cynomolgus macaques (Macaca fascicularis) with MPXV or CPXV, respectively [41-43].

\section{Conclusion}

To our knowledge, this study represents the first description of changes of the host cell gene expression program in response to infection with CPXV, a virus that is circulating in Europe as well as in parts of Asia and that displays some individual features in the genus OPV. While the host cell response towards VACV infection has been analysed in great detail by previous studies, we describe one of the first attempts to directly compare the impact of CPXV, MPXV and VACV on the gene expression profile of the host cell. We could show a major non-responsiveness of the transcriptional program of the host cell towards infection with all three viruses, which may be a sign of successful virostealth. In spite of this, we could also identify several genes which seemed to be affected by OPV infection in general or exhibited regulation by infection with a specific virus. By assigning these genes to certain biological processes or pathways, we could show that CPXV and MPXV infection induces several genes which are involved in immunity. This may indicate that CPXV and MPXV infection, but not VACV infection, induce a pronounced inflammatory response which may result in attraction of leukocytes. This leads to the question whether the induction of those genes is a part of the antiviral activity of the host or a process in benefit of infection as a mechanism that facilitates viral spread. The latter may be suggested by the important role which infected leukocytes are known to play in OPV dissemination across the body [27,44]. It will be interesting to analyse the effect of different viral strains or cell types on the observed effects, especially concerning the large genetic diversity of different CPXV strains or of VACV strains from different geographical origin.

\section{Methods}

\section{Cells and culture conditions}

All cell lines were obtained from American Type Culture Collection (ATCC, Manassas, VA). HeLa cells (ATCC ID CCL-2) were cultivated in Eagle's minimal essential medium (EMEM) supplemented with 10\% heat-inactivated foetal calf serum (FCS, PAA) and $2 \mathrm{mM}$ of L-glutamine (PAA). Hep-2 cells (ATCC ID CCL-23) and Vero E6 cells (ATCC ID CRL-1586) were both cultured in Dulbecco's Modified Eagle Medium (DMEM) containing 10\% FCS and $2 \mathrm{mM}$ of L-glutamine. All cell lines were routinely screened for the absence of mycoplasma contamination.

\section{Viruses and infection conditions}

VACV strain IHD-W (ATCC ID VR-1441, NCBI GenBank KC201194) and CPXV strain Brighton Red (ATCC ID VR-302; NCBI GenBank AF482758) were obtained from ATCC (ATCC, Manassas, VA). MPXV strain MSF-6 (sequence to be submitted soon), which was obtained from a fatally infected human in Congo, was kindly provided by Prof. Dr. Hermann Meyer (Institut für Mikrobiologie der Bundeswehr, München, Germany) [28]. All viruses were propagated in Hep-2 cells and cell culture supernatants which were clarified by centrifugation were used as virus stocks. The titres of virus stocks were determined by plaque assay [45] in Vero E6 cells as described before [46] and were shown to be comparable for CPXV, VACV and MPXV. All virus stocks were screened for absence of mycoplasma contamination. For infection experiments, HeLa cells were grown in $25 \mathrm{~cm}^{2}$ cell culture flasks (Nunc) and incubated overnight before infection with each virus at a multiplicity of infection of $5 \mathrm{PFU} / \mathrm{cell}$. Mock infections were performed using culture medium free of any virus. After adsorption of virus for $1 \mathrm{~h}$ at $37^{\circ} \mathrm{C}$, the virus-containing medium or mock medium was removed and cells were washed twice with phosphate buffered saline (PBS) to reduce side effects caused by biological factors probably present in the inoculum. Afterwards, fresh culture medium was added. Cells were then incubated at $37^{\circ} \mathrm{C}$ until $6 \mathrm{~h}$ post infection. All infection experiments were performed in biosafety level 3 (S3) laboratories in accordance with the German legal regulations.

\section{Sample acquisition and RNA preparation for microarray analysis}

Total RNA was isolated using Trizol ${ }^{\circledR}$ Reagent (Invitrogen) as described in the manufacturer's protocol for adherent cells. RNA samples from two independently infected cell cultures were used for each analysis. Human total RNA quality and integrity were determined using the Agilent 
RNA 6000 Nano Kit on the Agilent 2100 Bioanalyzer (Agilent Technologies). RNA was quantified by measuring A260 nm on the ND-1000 Spectrophotometer (NanoDrop Technologies). Sample labelling was performed as detailed in the Agilent "One-Color Microarray-Based Gene Expression Analysis" protocol (version 5.7, part number G414090040). Briefly, $1 \mu \mathrm{g}$ of each total RNA sample was used for the amplification and labelling step, using the Agilent Quick Amp Labeling Kit (Agilent Technologies) in the presence of cyanine 3-CTP. Yields of cRNA and the dyeincorporation rate were measured with the ND-1000 Spectrophotometer (NanoDrop Technologies).

\section{Microarrays and hybridisation}

The hybridisation procedure was performed according to the "One-Color Microarray-Based Gene Expression Analysis" protocol (Agilent Technologies, version 5.7, part number G4140-90040). Briefly, $1.65 \mu \mathrm{g}$ of Cy3-labeled fragmented cRNA in hybridisation buffer was hybridised overnight $\left(17 \mathrm{~h}, 65^{\circ} \mathrm{C}\right)$ to Agilent Whole Human Genome Oligo Microarrays $4 \times 44 \mathrm{~K}$, using Agilent's recommended hybridisation chamber and oven. Following hybridisation, the microarrays were washed once with the Agilent Gene Expression Wash Buffer 1 for $1 \mathrm{~min}$ at room temperature, followed by a second wash with preheated Agilent Gene Expression Wash Buffer $2\left(37^{\circ} \mathrm{C}\right)$ for $1 \mathrm{~min}$. The last washing step was performed with acetonitrile.

\section{Scanning and data analysis}

Fluorescence signals of the hybridised Agilent Microarrays were detected using Agilent's Microarray Scanner System (Agilent Technologies). The Agilent Feature Extraction Software (FES) 10.5.1.1 was used to read out and process the microarray image files. For determination of differential gene expression, FES-derived output data files were further analysed using the Rosetta Resolver gene expression data analysis system (Rosetta Biosoftware, Rosetta error model [47]). Ratios were calculated by dividing sample signal intensity through control signal intensity. The signal intensities from the single-experiment raw data lists were normalised by dividing the intensity values by their median. Putative candidate genes were selected based on a minimum fold change (FC) $\geq 2$ and p-value $\leq 0.01$. The calculation of merged ratios for replicate experiments was performed by calculating pair-wise log-ratios and log-ratio error and the subsequent combination to one ratio in an error-weighted averaging procedure. Data visualisation was done via generation of heat maps from the normalised signal intensity data using the software environment $R$ (v2.15.0).

\section{Pathway analysis}

Analysis of enrichment of genes to certain canonical pathways was done using the software Cytoscape 2.8.2 in combination with the Cytoscape plugin ClueGO v1.4 $[48,49]$. Enrichment analysis was based on terms provided by the KEGG databases (received $7^{\text {th }}$ February 2012) [50]. The probability of random association between the genes in the data set and the canonical pathway was calculated with a right-sided minimum likelihood test on the hypergeometric distribution using the Bonferroni correction of $\mathrm{p}$-values. Pathways that were identified with statistical support of p-values $\leq 0.05$ were taken into account. Due to the multitude of pathways which were identified by this method when analysing all differentially regulated genes, we decided to focus on significantly overrepresented terms. These were characterized by $\mathrm{p}$-values $\leq 0.05$, a ratio of $\geq 10 \%$ of term-annotated genes and a minimum of at least 4 genes per term.

\section{Cluster analysis}

Analysis of enrichment of genes to certain GO terms was also done using ClueGO. Enrichment analysis was based on terms provided by the GO (database file received $6^{\text {th }}$ February 2012) [51]. Again, we decided to focus on significantly overrepresented terms which were characterized by p-values $\leq 0.01$, a ratio of $\geq 10 \%$ of termannotated genes and a minimum of at least 6 genes per term. Furthermore, related terms were merged into functional groups and the most significant term of the group was defined as group leading term. The degree of connectivity between terms is calculated using $\mathrm{K}$ statistics and the calculated $\mathrm{K}$ score is also used for defining functional groups [48].

\section{Microarray data}

Microarray data have been submitted to the Gene Expression Omnibus (GEO) and can be searched using the record ID: GSE36854.

\section{Additional files}

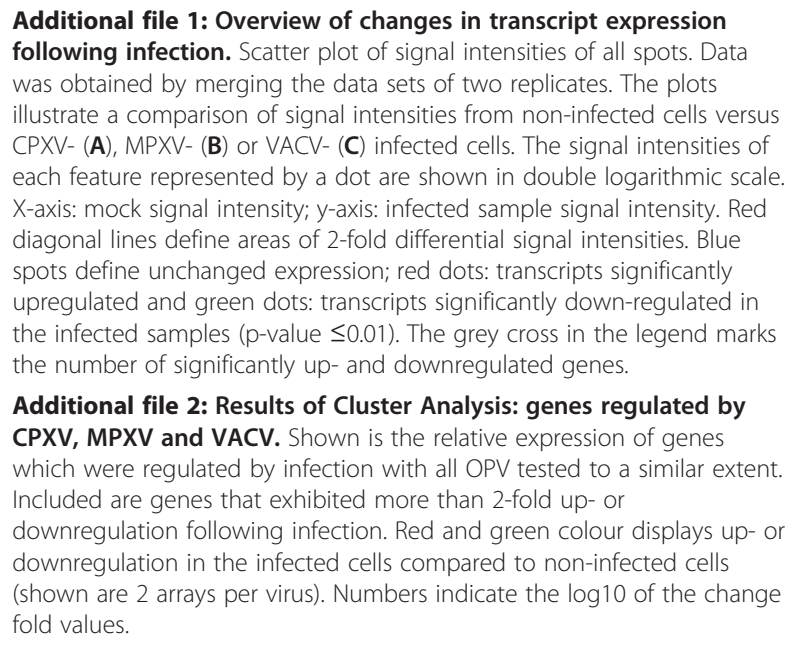


Additional file 3: Distribution of up- or downregulated genes. The figure shows the distribution of up- or downregulated transcripts following infection with CPXV (A), MPXV (B) or VACV (C) in comparison to non-infected cells. The degree of regulation compared to the noninfected control is displayed on the $x$-axis. A global minor

downregulation below the set cut-off value of $\geq 2$ fold change occurred following infection with MPXV and VACV.

\section{Competing interests}

The authors declare that they have no competing interests.

\section{Authors' contributions}

DB was responsible for design, conduct and completion of this work as well as for data analysis and writing of this manuscript. PWD was responsible for data processing and statistical analysis of microarray data. AN was the principal investigator and was primarily responsible for all aspects of research design and coordination and contributed to drafting the manuscript. All authors read and approved the final manuscript.

\section{Acknowledgements}

The authors would like to thank Jung-Won Sim-Brandenburg and Jule Hinzmann for excellent technical assistance and Ursula Erikli for copy-editing. Furthermore, we would like to thank Prof. Dr. Hermann Meyer for providing the MPXV strain used in this work.

This research was supported by the Robert Koch Institute and by the German National Academic Foundation.

\section{Author details}

${ }^{1}$ Centre for Biological Threats and Special Pathogens 1, Robert Koch Institute, Nordufer 20, Berlin 13353, Germany. ${ }^{2}$ Central Administration 4 (IT), Robert Koch Institute, Nordufer 20, Berlin 13353, Germany.

Received: 15 August 2012 Accepted: 8 February 2013

Published: 20 February 2013

\section{References}

1. Moss B: Poxviridae: the viruses and their replication. In Fields Virology. 5th edition. Edited by Knipe DM. Philadelphia: Lippincott Williams \& Wilkins; 2007:2905-2946.

2. International Committee on Taxonomy of Viruses, King AMQ: Virus taxonomy: classification and nomenclature of viruses: ninth report of the International Committee on Taxonomy of Viruses. London: Waltham, MA Academic Press; 2012.

3. Essbauer S, Pfeffer M, Meyer H: Zoonotic poxviruses. Vet Microbiol 2010, 140:229-236.

4. Fenner F, Anderson DA, Arita I, Jezek Z, Ladnyi ID: Smallpox and Its Eradication. Geneva: World Health Organization; 1988.

5. CDC: Laboratory-acquired vaccinia exposures and infections--United States, 2005-2007. MMWR Morb Mortal Wkly Rep 2008, 57:401-404.

6. Mercer AA, Schmidt A, Weber OF: Poxviruses. Basel Boston: Birkhäuser; 2007

7. de Souza TG, Drumond BP, Guedes MI, Leite JA, Mota BE, Campos MA, da Fonseca FG, Nogueira ML, Lobato ZI, Bonjardim CA, et al: Zoonotic vaccinia virus infection in Brazil: clinical description and implications for health professionals. J Clin Microbiol 2007, 45:1370-1372.

8. Trindade GS, Emerson GL, Carroll DS, Kroon EG, Damon IK: Brazilian vaccinia viruses and their origins. Emerg Infect Dis 2007, 13:965-972.

9. Trindade GS, Guedes MI, Drumond BP, Mota BE, Abrahao JS, Lobato Z Gomes JA, Correa-Oliveira R, Nogueira ML, Kroon EG, da Fonseca FG: Zoonotic vaccinia virus: clinical and immunological characteristics in a naturally infected patient. Clin Infect Dis 2009, 48:e37-e40.

10. Trindade GS, Lobato ZI, Drumond BP, Leite JA, Trigueiro RC, Guedes MI, da Fonseca FG, dos Santos JR, Bonjardim CA, Ferreira PC, Kroon EG: Short report: Isolation of two vaccinia virus strains from a single bovine vaccinia outbreak in rural area from Brazil: Implications on the emergence of zoonotic orthopoxviruses. Am J Trop Med Hyg 2006, 75:486-490.

11. Czerny CP, Eis-Hubinger AM, Mayr A, Schneweis KE, Pfeiff B: Animal poxviruses transmitted from cat to man: current event with lethal end. Zentralbl Veterinarmed B 1991, 38:421-431.
12. Pelkonen PM, Tarvainen K, Hynninen A, Kallio ER, Henttonen K, Palva A, Vaheri A, Vapalahti O: Cowpox with severe generalized eruption, Finland. Emerg Infect Dis 2003, 9:1458-1461.

13. Vorou RM, Papavassiliou VG, Pierroutsakos IN: Cowpox virus infection: an emerging health threat. Curr Opin Infect Dis 2008, 21:153-156.

14. Baxby D, Bennett M, Getty B: Human cowpox 1969-93: a review based on 54 cases. Br J Dermatol 1994, 131:598-607.

15. Breman JG, Kalisa R, Steniowski MV, Zanotto E, Gromyko Al, Arita I: Human monkeypox, 1970-79. Bull World Health Organ 1980, 58:165-182.

16. Chen N, Li G, Liszewski MK, Atkinson JP, Jahrling PB, Feng Z, Schriewer J, Buck C, Wang $C$, Lefkowitz $E$, et al: Virulence differences between monkeypox virus isolates from West Africa and the Congo basin. Virology 2005, 340:46-63.

17. Likos AM, Sammons SA, Olson VA, Frace AM, Li Y, Olsen-Rasmussen M, Davidson W, Galloway R, Khristova ML, Reynolds MG, et al: A tale of two clades: monkeypox viruses. J Gen Virol 2005, 86:2661-2672.

18. Arita I, Gispen R, Kalter SS, Wah LT, Marennikova SS, Netter R, Tagaya I: Outbreaks of monkeypox and serological surveys in nonhuman primates. Bull World Health Organ 1972, 46:625-631.

19. Guerra S, Lopez-Fernandez LA, Conde R, Pascual-Montano A, Harshman K, Esteban M: Microarray analysis reveals characteristic changes of host cell gene expression in response to attenuated modified vaccinia virus Ankara infection of human HeLa cells. J Virol 2004, 78:5820-5834.

20. Ludwig H, Mages J, Staib C, Lehmann MH, Lang R, Sutter G: Role of viral factor E3L in modified vaccinia virus ankara infection of human HeLa Cells: regulation of the virus life cycle and identification of differentially expressed host genes. J Virol 2005, 79:2584-2596.

21. Yang Z, Bruno DP, Martens CA, Porcella SF, Moss B: Simultaneous highresolution analysis of vaccinia virus and host cell transcriptomes by deep RNA sequencing. Proc Natl Acad Sci USA 2010, 107:11513-11518.

22. Brum LM, Lopez MC, Varela JC, Baker HV, Moyer RW: Microarray analysis of A549 cells infected with rabbitpox virus (RPV): a comparison of wild-type RPV and RPV deleted for the host range gene, SPI-1. Virology 2003, 315:322-334.

23. Guerra S, Lopez-Fernandez LA, Pascual-Montano A, Munoz M, Harshman K Esteban M: Cellular gene expression survey of vaccinia virus infection of human HeLa cells. J Virol 2003, 77:6493-6506.

24. Guerra S, Najera JL, Gonzalez JM, Lopez-Fernandez LA, Climent N, Gatell JM, Gallart T, Esteban M: Distinct gene expression profiling after infection of immature human monocyte-derived dendritic cells by the attenuated poxvirus vectors MVA and NYVAC. J Virol 2007, 81:8707-8721.

25. Langland JO, Kash JC, Carter V, Thomas MJ, Katze MG, Jacobs BL: Suppression of proinflammatory signal transduction and gene expression by the dual nucleic acid binding domains of the vaccinia virus E3L proteins. J Virol 2006, 80:10083-10095.

26. Alkhalil A, Hammamieh R, Hardick J, Ichou MA, Jett M, Ibrahim S: Gene expression profiling of monkeypox virus-infected cells reveals novel interfaces for host-virus interactions. Virol J 2010, 7:173.

27. Rubins KH, Hensley LE, Relman DA, Brown PO: Stunned silence: gene expression programs in human cells infected with monkeypox or vaccinia virus. PLoS One 2011, 6:e15615

28. Meyer H, Perrichot M, Stemmler M, Emmerich P, Schmitz H, Varaine F, Shungu R, Tshioko F, Formenty P: Outbreaks of disease suspected of being due to human monkeypox virus infection in the Democratic Republic of Congo in 2001. J Clin Microbiol 2002, 40:2919-2921.

29. Chang PY, Pogo BG: Reintroduction of gene(s) into an attenuated deletion mutant of vaccinia virus strain IHD-W. Microb Pathog 1993, 15:347-357.

30. Rubins KH, Hensley LE, Bell GW, Wang C, Lefkowitz EJ, Brown PO, Relman DA: Comparative analysis of viral gene expression programs during poxvirus infection: a transcriptional map of the vaccinia and monkeypox genomes. PLoS One 2008, 3:e2628.

31. Hanafusa H, Torii S, Yasunaga T, Nishida E: Sprouty1 and Sprouty2 provide a control mechanism for the Ras/MAPK signalling pathway. Nat Cell Biol 2002, 4:850-858

32. Impagnatiello MA, Weitzer S, Gannon G, Compagni A, Cotten M, Christofori G: Mammalian sprouty- 1 and -2 are membrane-anchored phosphoprotein inhibitors of growth factor signaling in endothelial cells. J Cell Biol 2001, 152:1087-1098.

33. Wakioka T, Sasaki A, Kato R, Shouda T, Matsumoto A, Miyoshi K, Tsuneoka M, Komiya S, Baron R, Yoshimura A: Spred is a Sprouty-related suppressor of Ras signalling. Nature 2001, 412:647-651. 
34. Zhang Z, Kobayashi S, Borczuk AC, Leidner RS, Laframboise T, Levine AD, Halmos B: Dual specificity phosphatase 6 (DUSP6) is an ETS-regulated negative feedback mediator of oncogenic ERK signaling in lung cancer cells. Carcinogenesis 2010, 31:577-586.

35. Kovanen PE, Rosenwald A, Fu J, Hurt EM, Lam LT, Giltnane JM, Wright G, Staudt LM, Leonard WJ: Analysis of gamma c-family cytokine target genes. Identification of dual-specificity phosphatase 5 (DUSP5) as a regulator of mitogen-activated protein kinase activity in interleukin-2 signaling. J Biol Chem 2003, 278:5205-5213.

36. Silva PN, Soares JA, Brasil BS, Nogueira SV, Andrade AA, de Magalhaes JC, Bonjardim MB, Ferreira PC, Kroon EG, Bruna-Romero O, Bonjardim CA: Differential role played by the MEK/ERK/EGR-1 pathway in orthopoxviruses vaccinia and cowpox biology. Biochem J 2006, 398:83-95.

37. Centers for Disease C, Prevention: Update: multistate outbreak of monkeypox--Illinois, Indiana, Kansas, Missouri, Ohio, and Wisconsin, 2003. MMWR Morb Mortal Wkly Rep 2003, 52:642-646.

38. Reynolds MG, Damon IK: Outbreaks of human monkeypox after cessation of smallpox vaccination. Trends Microbiol 2012, 20(2):80-87.

39. de Freitas MH, de Oliveira LC, Mugge FL, Ferreira PC, Trindade Gde S, Kroon EG, Bonjardim CA: The interplay between Aracatuba virus and host signaling pathways: role of PI3K/Akt in viral replication. Arch Virol 2011, 156:1775-1785.

40. Soares JA, Leite FG, Andrade LG, Torres AA, De Sousa LP, Barcelos LS, Teixeira MM, Ferreira PC, Kroon EG, Souto-Padron T, Bonjardim CA: Activation of the PI3K/Akt pathway early during vaccinia and cowpox virus infections is required for both host survival and viral replication. J Virol 2009, 83:6883-6899.

41. Johnson RF, Dyall J, Ragland DR, Huzella L, Byrum R, Jett C, St Claire M, Smith AL, Paragas J, Blaney JE, Jahrling PB: Comparative analysis of monkeypox virus infection of cynomolgus macaques by the intravenous or intrabronchial inoculation route. J Virol 2011, 85:2112-2125.

42. Johnson RF, Yellayi S, Cann JA, Johnson A, Smith AL, Paragas J, Jahrling PB, Blaney JE: Cowpox virus infection of cynomolgus macaques as a model of hemorrhagic smallpox. Virology 2011, 418:102-112.

43. Smith AL, St Claire M, Yellayi S, Bollinger L, Jahrling PB, Paragas J, Blaney JE, Johnson RF: Intrabronchial inoculation of cynomolgus macaques with cowpox virus. J Gen Virol 2012, 93:159-164.

44. Wahl-Jensen V, Cann JA, Rubins KH, Huggins JW, Fisher RW, Johnson AJ, de Kok-Mercado F, Larsen T, Raymond JL, Hensley LE, Jahrling PB: Progression of pathogenic events in cynomolgus macaques infected with variola virus. PLOS One 2011, 6:e24832.

45. Tsuchiya $Y$, Tagaya I: Plaque assay of variola virus in a cynomolgus monkey kidney cell line. Arch Gesamte Virusforsch 1970, 32:73-81.

46. Witkowski PT, Schuenadel L, Wiethaus J, Bourquain DR, Kurth A, Nitsche A: Cellular impedance measurement as a new tool for poxvirus titration, antibody neutralization testing and evaluation of antiviral substances. Biochem Biophys Res Commun 2010, 401:37-41.

47. Weng L, Dai H, Zhan Y, He Y, Stepaniants SB, Bassett DE: Rosetta error model for gene expression analysis. Bioinformatics 2006, 22:1111-1121.

48. Bindea G, Mlecnik B, Hackl H, Charoentong P, Tosolini M, Kirilovsky A, Fridman WH, Pages F, Trajanoski Z, Galon J: ClueGO: a Cytoscape plug-in to decipher functionally grouped gene ontology and pathway annotation networks. Bioinformatics 2009, 25:1091-1093.

49. Smoot ME, Ono K, Ruscheinski J, Wang PL, Ideker T: Cytoscape 2.8: new features for data integration and network visualization. Bioinformatics 2011, 27:431-432.

50. Kanehisa M, Goto S: KEGG: kyoto encyclopedia of genes and genomes. Nucleic Acids Res 2000, 28:27-30.

51. Ashburner M, Ball CA, Blake JA, Botstein D, Butler H, Cherry JM, Davis AP, Dolinski K, Dwight SS, Eppig JT, et al: Gene ontology: tool for the unification of biology. The Gene Ontology Consortium. Nat Genet 2000, 25:25-29.

doi:10.1186/1743-422X-10-61

Cite this article as: Bourquain et al:: Comparison of host cell gene expression in cowpox, monkeypox or vaccinia virus-infected cells reveals virus-specific regulation of immune response genes. Virology Journal 2013 10:61.

\section{Submit your next manuscript to BioMed Central and take full advantage of:}

- Convenient online submission

- Thorough peer review

- No space constraints or color figure charges

- Immediate publication on acceptance

- Inclusion in PubMed, CAS, Scopus and Google Scholar

- Research which is freely available for redistribution

Submit your manuscript at www.biomedcentral.com/submit
C BioMed Central 